\title{
circSPG21 protects against intervertebral disc disease by targeting miR-1197/ATP1B3
}

\author{
Yizhen Huang ${ }^{1,2,5}$, Zhenlei Zhang ${ }^{1,2,5}$, Jianle Wang ${ }^{1,2,5}$, Shuying Shen $\mathbb{D}^{1,2}$, Teng Yao ${ }^{1,2}$, Yining X ${ }^{3}$, Zizheng Chen ${ }^{1,2}$, Bin Fang ${ }^{4 凶}$ and \\ Jianjun $\mathrm{Ma}^{1,2 \mathrm{~m}}$
}

(c) The Author(s) 2021

The abnormal expression of circular RNAs (circRNAs) is associated with numerous human diseases. This study investigated the mechanism by which circRNA acts as competitive endogenous RNA in the regulation of degenerative intervertebral disc disease (IVDD). Decreased expression of circSPG21 was detected in degenerated nucleus pulposus cells (NPCs), the function of circSPG21 in NPCs was explored and verified, and the downstream target of circSPG21 was investigated. The interaction between circSPG21 and miR-1197 and its target gene (ATP1B3) was studied by online database prediction and molecular biological verification. Finally, the circSPG21/miR-1197/ATP1B3 axis was verified in the mouse tail-looping model. The expression of circSPG21 in the nucleus pulposus in IVDD was directly related to an imbalance of anabolic and catabolic factors, which affected cell senescence. circSPG21 was found to play a role in human NPCs by acting as a sponge of miR-1197 and thereby affecting ATP1B3. The regulation of circSPG21 provides a potentially effective therapeutic strategy for IVDD.

Experimental \& Molecular Medicine (2021) 53:1547-1558; https://doi.org/10.1038/s12276-021-00674-z

\section{INTRODUCTION}

Degenerative disc disease is an age-related disease ${ }^{1}$. With increasing age, the disc gradually loses flexibility, elasticity, and shock absorption function, and the fibrous rings that surround the disc become fragile and tear easily ${ }^{2}$. Simultaneously, the central nucleus pulposus (NP) loses water and atrophies. Lower back pain caused by degenerative changes in the intervertebral disc is an important reason for the decline and loss of the working ability of the population, which has severe social and economic impacts. The current clinical treatment strategy for this disease primarily focuses on alleviating symptoms and cannot prevent or treat disc degeneration ${ }^{3}$. Therefore, an indepth understanding of the mechanism of disc degeneration has crucial scientific value and could provide a theoretical basis for identifying new prevention and treatment strategies for disc degeneration ${ }^{4}$.

In contrast to traditional linear RNA (including $5^{\prime}$ and $3^{\prime}$ ends), circular RNA (circRNA) has a closed ring structure that is unaffected by RNA exonucleases, is more stably expressed, and is not easily degraded ${ }^{5}$. Some circRNAs have microRNA (miRNA) response elements and interact with miRNAs. Because of the high expression level and stability of circRNA, it is used as a competitive endogenous RNA (ceRNA) ${ }^{6}$. circRNA competes with miRNA to prevent the posttranslational inhibition of miRNA and target coding RNA, ultimately regulating the expression levels of target genes? .

In a study of intervertebral disc degeneration, circVMA21 was found to act as a sponge of miR-200c to regulate X-linked inhibitor-of-apoptosis protein (XIAP) and affected cell apoptosis and the imbalance of extracellular matrix anabolism and catabolism. Thus, circVMA21 could be used as a prognostic biomarker of intervertebral disc disease (IVDD) and a potential therapeutic target ${ }^{8}$. In a study of the functional mechanism of circRNA in the development of intervertebral disc degeneration, circERCC2 was found to alleviate IVDD by targeting the miR-182$5 \mathrm{p} / \mathrm{SIRT} 1$ axis; it is therefore now used as a prognostic marker and therapeutic target for IVDD 9 .

miRNAs are noncoding single-stranded RNA molecules of $\sim 22$ nucleotides encoded by endogenous genes ${ }^{10}$. They promote target gene degradation or posttranscriptionally inhibit translation. miR-199a-5p promotes NP cell apoptosis and IVDD by inhibiting SIRT1-dependent p21 deacetylation, and miR-199a-5p might thus be an important therapeutic target for IVDD ${ }^{11}$. miR$532-3 p$ inhibits nonsmall cell lung cancer (NSCLC) via forkhead Box P3 (FOXP3), suggesting that the miR-532-3p/FOXP3 axis could be a potential therapeutic target ${ }^{12}$. At present, T-box transcription factor 1 (TBX1) is considered one of the core genes related to congenital heart disease, and miR-144 plays an inhibitory role in cardiomyocytes through the TBX1/Janus kinase 2/signal transducer and activator of transcription 1 axis ${ }^{13}$.

ATP1B3 encodes ATPase $\mathrm{Na}^{+} / \mathrm{K}^{+}$transporting subunit beta 3, a complete membrane protein that establishes and maintains the electrochemical gradient of $\mathrm{Na}^{+}$and $\mathrm{K}^{+}$ions across the plasma membrane. This protein is involved in transmembrane transport, maintaining neuromuscular excitation, and regulating osmotic pressure. As exercise changes the expression of

\footnotetext{
${ }^{1}$ Department of Orthopaedic Surgery, Sir Run Run Shaw Hospital, Zhejiang University School of Medicine, Zhejiang, China. ${ }^{2}$ Key Laboratory of Musculoskeletal System Degeneration and Regeneration Translational Research of Zhejiang Province, Zhejiang, China. ${ }^{3}$ Shaoxing University School of Medicine, Shaoxing, China. ${ }^{4}$ Department of Spine Surgery, Shaoxing Central Hospital, China Medical University, Shaoxing, China. ${ }^{5}$ These authors contributed equally: Yizhen Huang, Zhenlei Zhang, Jianle Wang

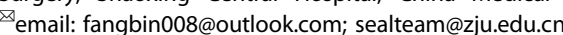


$\mathrm{Na}^{+} / \mathrm{K}^{+}$ATPase in skeletal muscle ${ }^{14}$ and the intervertebral disc is a load-bearing organ in the spine whereas NP tissue is rich in water, we hypothesize that $\mathrm{Na}^{+} / \mathrm{K}^{+}$ATPase is associated with disc degeneration.

In this study, a circRNA derived from the autosomal recessive spastic paraplegia (SPG21) gene ${ }^{15}$ (designated circSPG21; also referred to as hsa_circ_0035875 in CircBase (http://www. circbase.org)) was identified in the NP, and its role was systemically investigated in cell and animal models of IVDD. circSPG21 is rich in miRNA-binding sites and plays a role as a ceRNA. circSPG21 acts as a sponge of miR-1197, which regulates the target gene $A T P 1 B 3^{16}$. It was found that circRNA acts as ceRNA to regulate the activity and function of nucleus pulposus cells (NPCs) and the pathological process of IVDD.

\section{MATERIALS AND METHODS Human intervertebral disc tissue}

Human NP tissues were collected, preserved, and studied according to the protocols and guidelines of the Ethics Committee of Sir Run Shaw Hospital (Zhejiang, China). Control disc tissue was removed from patients with lumbar fractures who had no history of degeneration, and pathological tissue was removed from patients with disc herniation during disc replacement. All operations and risks were reported to the patient, and informed consent was provided prior to tissue collection. Patient information is included in the Supplementary Table $1^{17}$.

\section{Mouse disc degeneration model}

All mice were purchased from Shanghai SLAC Laboratory Animal Co., Ltd. (Shanghai, China). All animal experiments were performed in accordance with the principles and procedures of the National Institutes of Health Guide for the Care and Use of Laboratory Animals and the guidelines for animal treatment of Sir Run Run Shaw Hospital (Zhejiang University-affiliated, Hangzhou, Zhejiang). Six-week-old C57BL/6 mice were selected, and the tails of the mice were fixed with a thin wire after anesthesia. The tail was bent into a ring shape and held in place for one month to induce chronic degeneration of the intervertebral disc under simulated pressure ${ }^{18}$.

\section{Intraperitoneal injection of circSPG21}

circSPG21-wt and circSPG21-mut adenoviruses were constructed by Hanbio Biotechnology (Shanghai) Co. Ltd. After successful tail model generation, the mice were intraperitoneally injected with adenovirus solution once per week for 8 weeks. A $100 \mu \mathrm{L}$ solution containing circSPG21-wt or circSPG21mut was slowly injected into the abdominal cavity.

\section{NPC culture}

NPCs were isolated from humans and mice and cultured in Dulbecco's modified Eagle's medium containing 10\% fetal bovine serum (Thermo Fisher Scientific, Waltham, MA, USA). The cells were cultured in a humidified environment at $37{ }^{\circ} \mathrm{C}$ with $5 \% \mathrm{CO}_{2}$ and $95 \%$ air. To ensure stability, NPCs were used within three generations.

\section{Bioinformatics analysis}

Target microRNAs of circSPG21 were identified using two online databases (TargetScan: http://www.targetscan.org/and miRanda: http://www.microrna.org/) and RNAhybrid analysis software. Three online databases were used to predict target genes of miR-1197 (TargetScan, miRanda, and miRTarBase: http:// mirtarbase.mbc.nctu.edu.tw/php/download.php). The corresponding binding sites were identified using online software (Circlnteractome: https:// circinteractome.nia.nih.gov/index.html and TargetScan).

\section{RNA extraction and RT-qPCR analysis}

According to the manufacturer's instructions, total cellular RNA was extracted from cultured NPCs using TRIzol reagent (Invitrogen, Carlsbad, CA, USA). miRNA was extracted using an miRNA extraction kit (CWBIO, Beijing, China). RNA was stored at $-80^{\circ} \mathrm{C}$, and $1.0 \mu \mathrm{g}$ of total RNA was used for reverse transcription using an miRNA CDNA kit or a HiFiScript CDNA kit (CWBIO, Beijing, China) to study miRNA and mRNA expression.
The amplification reaction contained $1 \mu \mathrm{L}$ cDNA, $5 \mu \mathrm{L}$ SYBR Green Master Mix (Yeasen Biotech Co., Ltd, Shanghai, China), $1 \mu \mathrm{L}$ primer (TsingKe, Hangzhou, China), and $3 \mu \mathrm{L}$ water, for a total of $10 \mu \mathrm{L}$. The mixture was denatured at $95^{\circ} \mathrm{C}$ for $5 \mathrm{~s}$ and amplified at $60^{\circ} \mathrm{C}$ for $24 \mathrm{~s}(40$ cycles). An ABI 7500 Sequencing Detection System (Applied Biosystems, Foster City, CA, USA) was used to establish an amplification reaction in a $10 \mu \mathrm{L}$ volume containing amplification primers and Supermix (including ROX; CWBIO). Please refer to the Supplementary Table 2 for all primer sequences.

\section{X-ray analysis}

Samples were fixed in polyoxometalate and stored in $70 \%$ ethanol. The samples were scanned at $45 \mathrm{kV}$ and $50 \mathrm{~mA}$ for $5 \mathrm{~s}$, and the data were analyzed using ImageJ software (National Institutes of Health, Bethesda, MD, USA).

\section{Adenovirus overexpression}

To construct the recombinant adenovirus expressing circSPG21 under the control of the mouse cytomegalovirus (CMV) promoter, PCR-cloned circular gene CDNA (Ad-circSPG21) and the green fluorescent protein gene (Ad-GFP) were inserted into the pAdEasy-EF1-MCS-CMV-GFP vector (Han Biological, Shanghai, China). Cells were infected with the virus, and the amount of virus was adjusted according to the virus titer and the number of cells (virus amount $=$ multiplicity of infection $\times$ cell number/virus titer (plaque-forming units $/ \mathrm{mL}$ ) $\times 1000$ ). After $4 \mathrm{~h}$ of infection, the medium was changed, and green fluorescence was observed under a fluorescence microscope to determine whether overexpression was successful ${ }^{19}$.

\section{RNA interference and overexpression}

siRNA-mediated gene knockout suppressed circSPG21 expression. Three different siRNAs were designed and tested. Specific inhibitors and mimics (RiboBio, Guangzhou, China) were used to inhibit or induce the expression of miR-1197. Lipofectamine RNAiMAX transfection reagent (Thermo Fisher) was used to study siRNAs, miR-1197 inhibitors, and mimetics. NPCs were seeded in six-well plates, and transfection was performed after they covered $60-80 \%$ of the area. Then, $9 \mu \mathrm{L}$ of Lipofectamine RNAiMAX transfection reagent and $3 \mu \mathrm{L}$ of siRNA (or inhibitors, mimetics) were diluted with $150 \mu \mathrm{L}$ of Opti-MEM Medium each (Thermo Fisher). After the two dilutions were mixed, they were allowed to stand at room temperature for $5 \mathrm{~min}$. Next, $250 \mu \mathrm{L}$ of the mixture was added to the six-well plates for transfection. The cells were cultured at $37^{\circ} \mathrm{C}$ for $48 \mathrm{~h}$ and then used in the experiment.

\section{$\boldsymbol{\beta}$-galactosidase staining}

NPCs were stained with a senescence $\beta$-galactosidase staining kit (C0602, Beyotime, Shanghai, China). After fixing the cells, they were stained with mixed staining solution for $8 \mathrm{~h}\left(37^{\circ} \mathrm{C}\right)$. After removing excess dye, images were acquired under a microscope ${ }^{20}$.

\section{Alcian staining}

Alcian staining was performed using a kit (G1563, Solarbio, Beijing, China). NPCs were added to 24-well plates and fixed with formalin. After adding the acidification solution, the cells were stained for $30 \mathrm{~min}$. Excess dye was removed, and images were acquired using a scanner (V600, EPSON, Nagano, Japan) $)^{21}$.

\section{Western blotting}

The medium was removed from the wells, and the cells were washed with phosphate-buffered saline (PBS). Human or mouse NPCs were lysed using radioimmunoprecipitation assay buffer (Beyotime, China). The protein extract was separated using $10 \%$ sodium dodecyl sulfate-polyacrylamide gel electrophoresis, transferred to a polyvinylidene difluoride (PVDF) membrane (Bio-Rad, Hercules, CA, USA), and blocked with skim milk powder for $1 \mathrm{~h}$. The PVDF membrane was incubated with protein antibodies (1:1000, Abcam) at $4{ }^{\circ} \mathrm{C}$ overnight (collagen II, aggrecan, SOX9, MMP3, MMP13, ADAMTS4, and ADAMTS5). After washing with Tris-buffered saline with $0.1 \%$ Tween ${ }^{\circledR} 20$, the cells were further incubated with the secondary antibody at room temperature $\left(25^{\circ} \mathrm{C}\right)$ for $2 \mathrm{~h}$. Protein bands were observed using the LAS-4000 Science Imaging System (Fujifilm, Tokyo, Japan) and analyzed with ImageJ software (National Institutes of Health, Bethesda, MD, USA). 


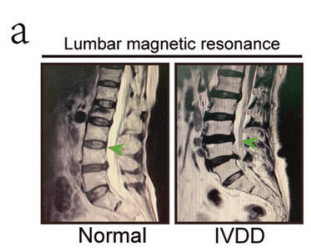

e

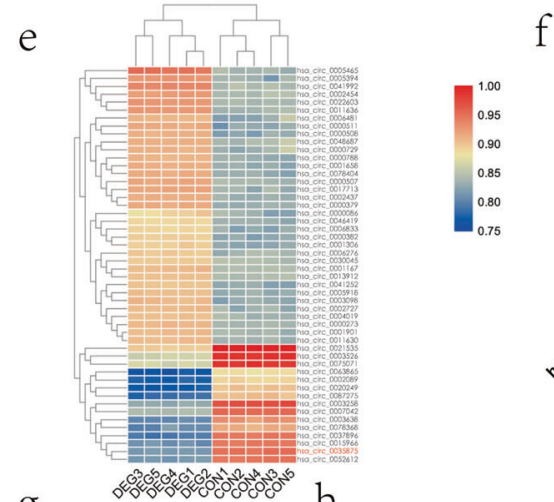

$g$
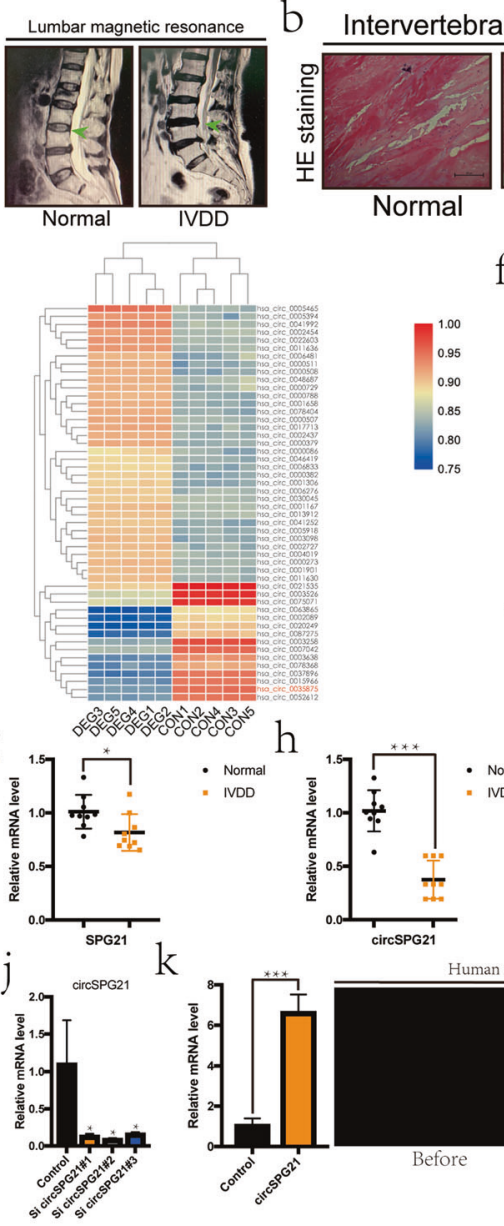

Normal
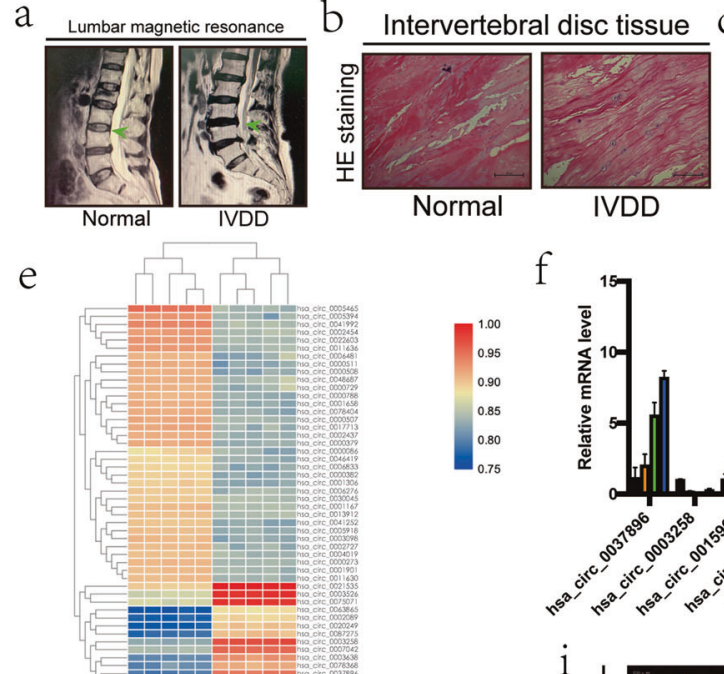

f
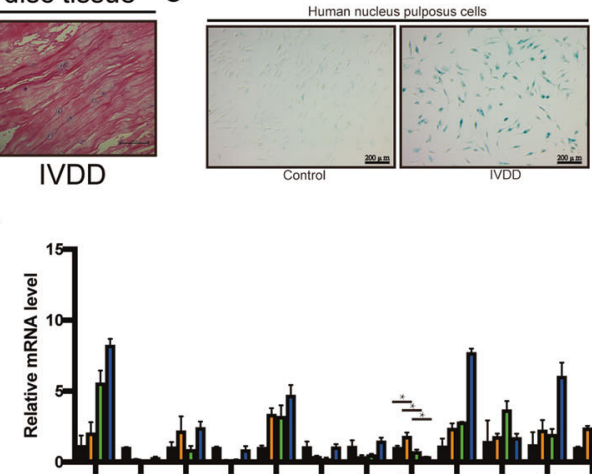

d
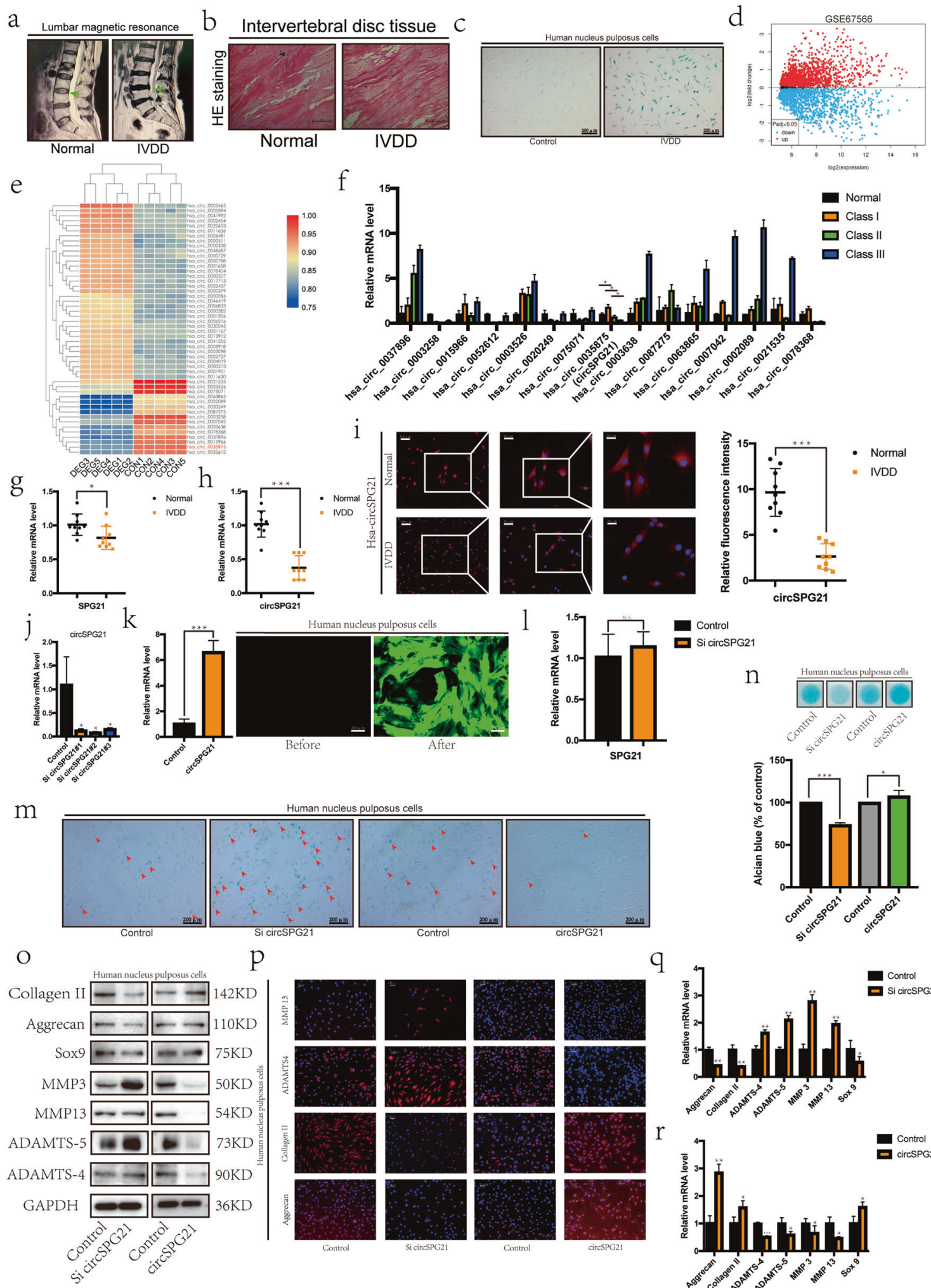

$\mathrm{p}$
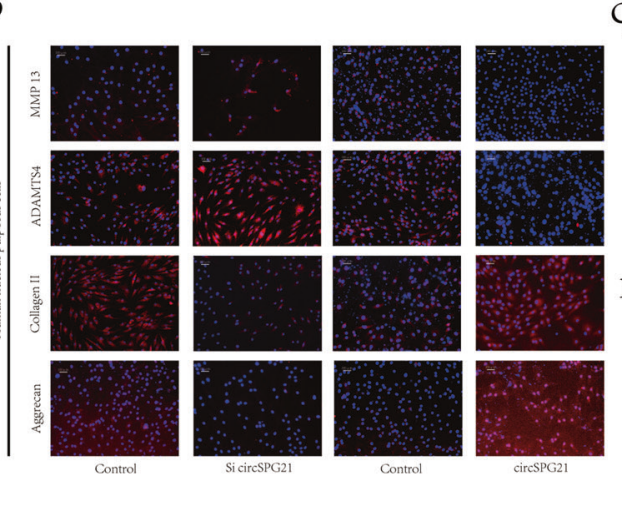

q
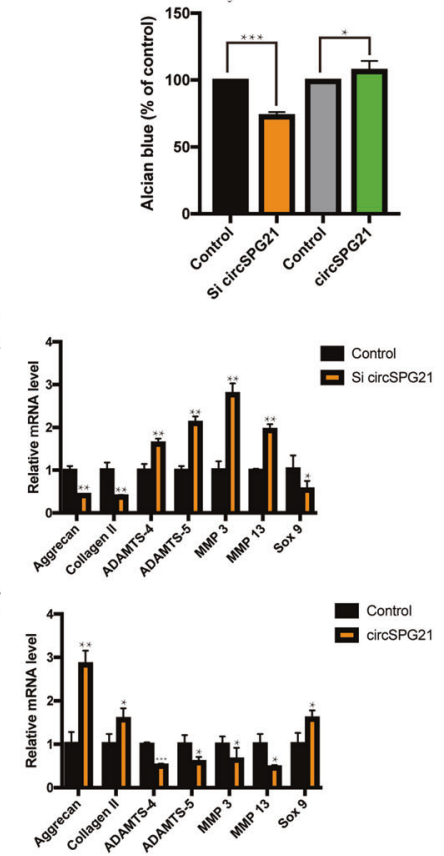

\section{Immunofluorescence}

NPCs were cultured in $20 \mathrm{~mm}$ glass-bottom cell culture dishes (801001; Nest Biotechnology Co., Ltd., Shanghai, China). NPCs were fixed with $4 \%$ paraformaldehyde for $20 \mathrm{~min}$. After washing with PBS, the cells were permeated with $0.3 \%$ Triton X-100 for 30 min and then blocked with $5 \%$ bovine serum albumin for $60 \mathrm{~min}$. Cells were then incubated overnight at $4{ }^{\circ} \mathrm{C}$ with collagen II, aggrecan, MMP3, or ADAMTS4 antibodies (1:200;
Abcam). The next day, the cells were washed with PBS and incubated with goat anti-rabbit IgG conjugated with the fluorescent dye Cy5 (1:100; Abcam). For nuclear staining, 4',6-diamidino-2-phenylindole (Life Technologies, Carlsbad, CA, USA) was used. Immunofluorescence images were obtained using Nikon Eclipse TI and Zeiss LSM780 confocal microscopes and processed using Image-Pro Plus 6.0 ( $\mathrm{NIH}$, Bethesda, MD, USA). 
Fig. 1 circSPG21 regulates NPC extracellular matrix (ECM) metabolism. a MRIs were compared between the normal and degenerative groups; $n=2$ (two different donors). b HE staining was performed in the normal and degenerative group samples (scale bar, $100 \mu \mathrm{m}$ ); $n=2$ (two different donors). $\mathrm{c}$ The degree of cell senescence was detected by $\beta$-galactosidase staining (scale bar, $200 \mu \mathrm{m}$ ); $n=2$ (two different donors). d A volcano map was drawn according to the GEO database (GSE67566). e Heat map of all differentially expressed circular RNAs (circRNAs) in the degenerated and control disc tissues. f After screening 15 circRNAs, the relationship between circRNAs and the severity of degeneration was determined by real-time quantitative polymerase chain reaction (RT-qPCR), $n=4$ (four different donors), ${ }^{*} p<0.05$. $\mathbf{g}$ The expression of SPG21, as measured by RT-qPCR, in human degenerated tissue differed significantly from that in the control group; $n=6$, ${ }^{*} p<$ 0.05. Data are presented as the mean \pm S.D., and the $p$ values were determined by a two-tailed unpaired Student's $t$-test. $\mathbf{h}$ The expression of circSPG21, as measured by RT-qPCR, in human degenerated tissue was significantly lower than that in control tissue; $n=6$, ${ }^{* * *} p<0.001$. Data are presented as the mean \pm S.D., and the $p$ values were determined by a two-tailed unpaired Student's $t$-test. i Left, the expression of circSPG21, as measured by FISH analysis, in human degenerated tissue was lower than that in control tissue. Representative images (scale bar, 25-100 $\mu \mathrm{m}$ ) are displayed (two different donors). Right panel, the intensity of circSPG21 expression, as analyzed using fluorescence intensity, ${ }^{* * *} p<0.001$. Data are presented as the mean \pm S.D., and the $p$ values were determined by a two-tailed unpaired Student's $t$-test. $\mathbf{j}$ Nucleus pulposus cells (NPCs) were transfected with circSPG21 siRNA\# 1, 2, 3, or negative control siRNA (concentration $20 \mathrm{~nm}$ ). The expression level of circSPG21, as determined using RT-qPCR, ${ }^{*} p<0.05$. Data are presented as the mean \pm S.D., and the $p$ values were determined by a two-tailed unpaired Student's $t$-test. $\mathbf{k}$ circSPG21 overexpression in NPCs after adenovirus infection with circSPG21. Left, circSPG21 was highly expressed in NPCs. Right, after virus infection, green fluorescence was observed under a fluorescence microscope (scale bar, $200 \mu \mathrm{m})$; ${ }^{* * *} p<0.001$. Data are presented as the mean \pm S.D., and the $p$ values were determined by a two-tailed unpaired Student's $t$-test. I Expression of SPG21, as detected by RT-qPCR, after transfection with si-circSPG21. Data are presented as the mean \pm S.D., and the $p$ values were determined by a two-tailed unpaired Student's $t$-test. $\mathbf{m} \beta$-Galactosidase staining was performed to evaluate cell aging (scale bar, $200 \mu \mathrm{m}$ ). $\mathbf{n}$ Alcian staining was performed to evaluate the extracellular matrix; ${ }^{*} p<0.05,{ }^{* *} p<0.01$. Data are presented as the mean \pm S.D., and the $p$ values were determined by a two-tailed unpaired Student's $t$-test. o Expression of proteins (collagen II, aggrecan, SRY-box transcription factor 9 (SOX9), matrix metallopeptidase 3 (MMP3), MMP13, ADAM metallopeptidase with thrombospondin type 1 motif 4 (ADAMTS4), and ADAMTS5), as determined by western blotting. p Expression of collagen II, aggrecan, ADAMTS4, and MMP13, as determined by immunofluorescence (IF), compared according to the fluorescence intensity (Scale bar, $100 \mu \mathrm{m}$ ). Expression of genes after circSPG21 knockdown (q) or overexpression $(\mathbf{r})$, as measured by RT-qPCR; ${ }^{*} p<0.05,{ }^{* *} p<0.01$. Data are presented as the mean \pm S.D., and the $p$ values were determined by a two-tailed unpaired Student's $t$-test.

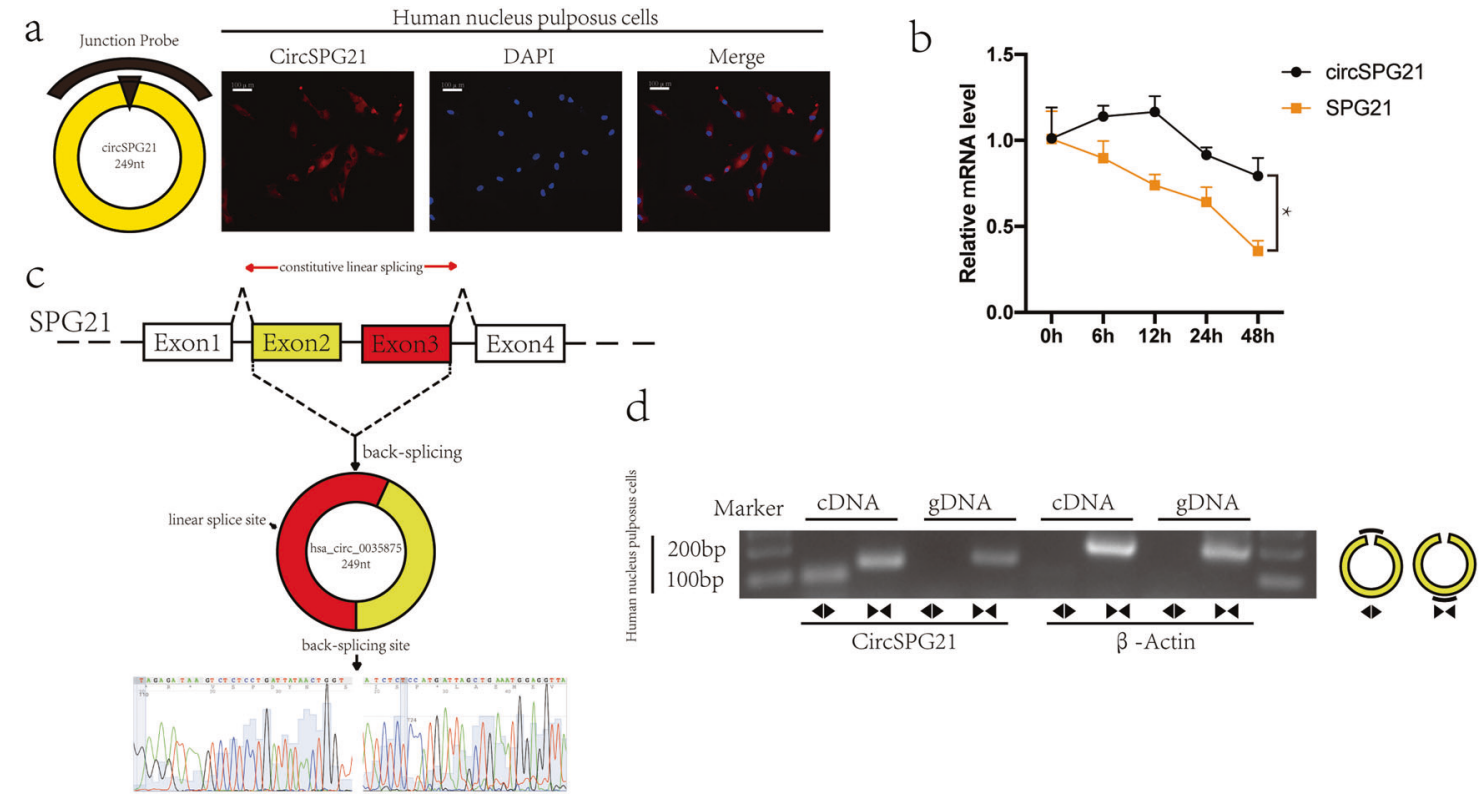

Fig. 2 Ring structure of circSPG21. a Left, circSPG21 and probe diagram. Right, RNA-fluorescence in situ hybridization (FISH) showed that circSPG21 was mainly present in the cytoplasm (scale bar, $100 \mu \mathrm{m}$ ). The circular RNA probe was labeled with Cy-3. Nuclei were stained with 4',6-diamidino-2-phenylindole (DAPI). b Nucleus pulposus cells were treated with $5 \mu \mathrm{g} / \mathrm{mL}$ actinomycin D, and the expression levels of circSPG21 and SPG21 were detected by RT-qPCR, ${ }^{*} p<0.05$. Data are presented as the mean \pm S.D., and the $p$ values were determined by a twotailed unpaired Student's $t$-test. c Exons 2-3 of SPG21 form circSPG21. Sanger sequencing confirmed the presence of circSPG21. d Left: circSPG21 was amplified by divergent and convergent primers in CDNA and genomic DNA and separated by horizontal electrophoresis. $\beta$-actin served as a negative control. Right: a diagram of the divergent and convergent primers.

\section{RNA FISH}

The Cy3-labeled circSPG21 probe and 488-labeled locked nucleic acid miR1197 probe were designed and synthesized by Haoke (Wuhan, China). A FISH kit (RiboBio, Guangzhou, China) was used to detect probe signals in human NPCs. Images were acquired using a Nikon A1Si laser scanning confocal microscope (Nikon Instruments Inc., Japan).

\section{Statistical analysis}

Statistical analysis was performed using SPSS v22.0. The unpaired data between the two groups were tested using the $t$-test (using the $95 \%$ confidence interval for differences between groups). Differences between groups with values of $p<0.05$ were considered significant. 
C

a

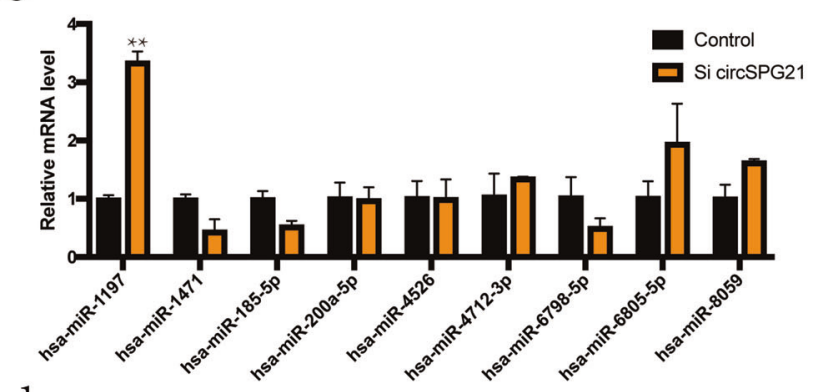

hsa-miR-1197

hsa-miR-1471

hsa-miR-185-5p

hsa-miR-200a-5p

hsa-miR-4526

hsa-miR-4712-3p

hsa-miR-6798-5p

hsa-miR-6805-5p

hsa-miR-8059 d

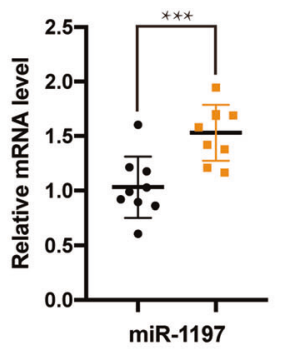

f
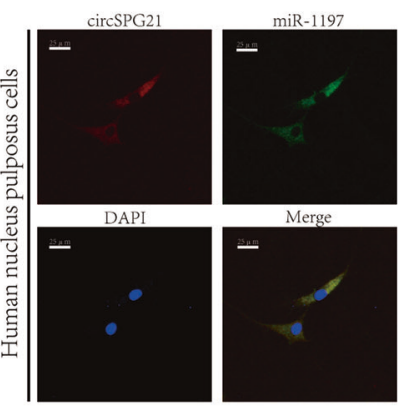

i

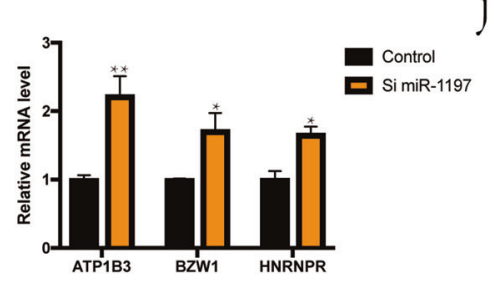

e

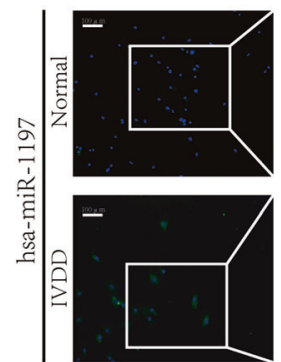

g

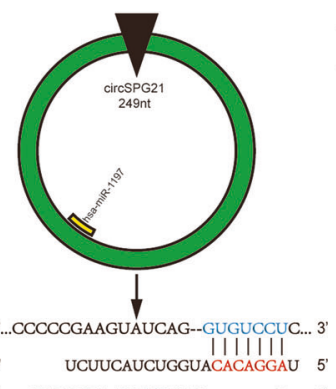

Mut 5, CCCCCGAGUAUCAG-8rgucou 3
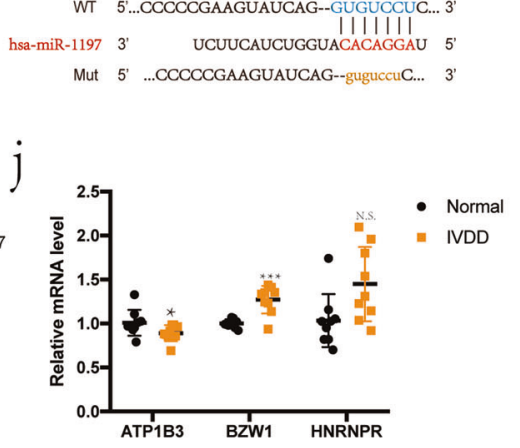

$\mathrm{h}$ b
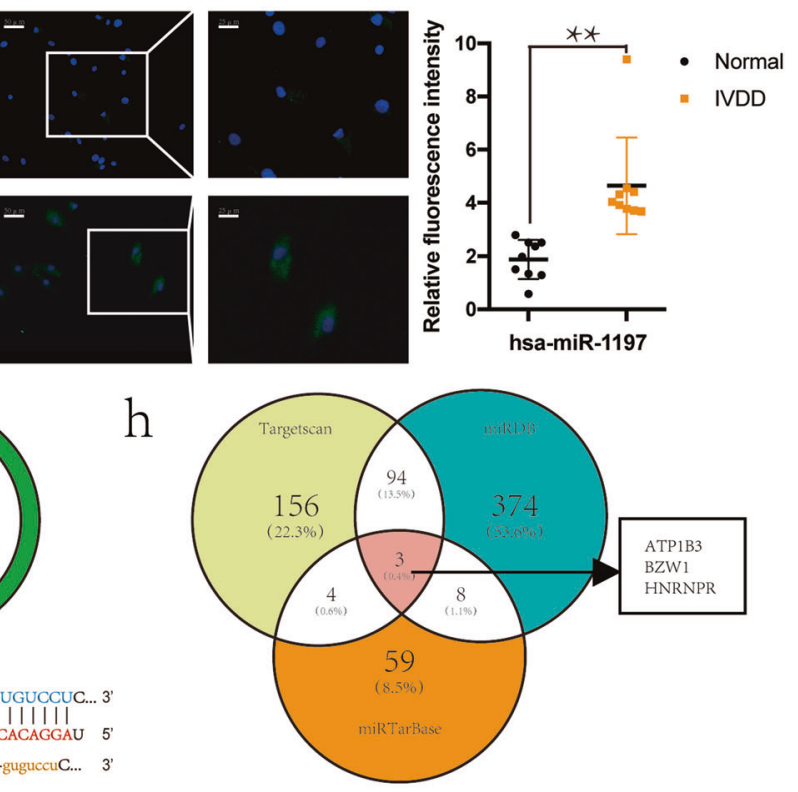

K

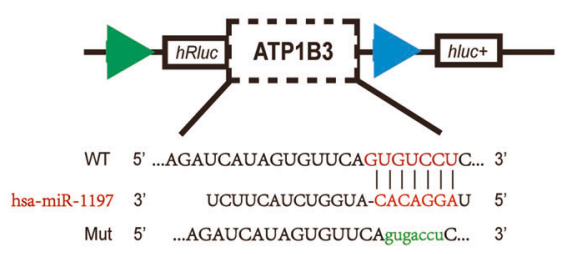

\section{RESULTS}

Comparison of normal and degenerative intervertebral discs In the clinical analysis, when a patient showed intervertebral disc degeneration, the high signal for the intervertebral disc in T2 MRI images disappeared, indicating water loss from the degenerative intervertebral disc (Fig. 1a). After obtaining consent from the patients, we collected the intervertebral discs of patients with spinal fracture due to trauma as the control group and those from patients with intervertebral disc degeneration as the degeneration group. After HE staining, the fibrous tissue in the degenerative group appeared loose compared with that in the control group, and the NPCs formed cell clusters (Fig. 1b). We extracted NPCs from the surgical specimens for $\beta$-galactosidase staining. Most cells in the degenerative group were green, indicating cell aging (Fig. 1c). Based on these results, when intervertebral disc degeneration occurs, a series of changes also occur in NP tissue and cells. The factor affecting the activity of NPCs could thus be a new target for treating disc degeneration.

circSPG21 is expressed at low levels in degenerative tissues In the study of RNAs, it was discovered that the interaction between circRNAs and miRNAs acts as a key regulator of gene 
Fig. 3 ceRNA network construction. a circSPG21-target microRNA was predicted based on the intersection of the TargetScan, miRanda, and RNAhybrid software data. b A ceRNA network diagram was drawn using Cytoscape. c Expression of microRNAs after circSPG21 knockdown, as detected by real-time quantitative polymerase chain reaction (RT-qPCR), ${ }^{* *} p<0.01$. Data are presented as the mean \pm S.D., and the $p$ values were determined by a two-tailed unpaired Student's $t$-test. d miR-1197 expression increased in degenerated tissues, as measured by RT-qPCR; $n=6,{ }^{* * *} p<0.001$. Data are presented as the mean \pm S.D., and the $p$ values were determined by a two-tailed unpaired Student's $t$-test. e Left, the expression of miR-1197, as measured by fluorescence in situ hybridization (FISH), in degenerated tissues was higher than that in control tissues (scale bar, $100 \mu \mathrm{m}$ ). Right panel, the fluorescence intensity of miR-1197 was stronger in degenerated tissue (two different donors), ${ }^{* *} p<$ 0.01 . Data are presented as the mean \pm S.D., and the $p$ values were determined by a two-tailed unpaired Student's $t$-test. The miR-1197 probe was labeled with Alexa Fluor 488. f circSPG21 and miR-1197 were localized in the cell, and both were seen in the cytoplasm (scale bar, $25 \mu \mathrm{m}$ ). g The sites where circSPG21 and miR-1197 bind to each other were identified using Circlnteractome. $\mathbf{h}$ The target genes of miR-1197 were predicted based on the intersection of TargetScan, miRDB, and miRTarBase results. i Expression of target genes after miR-1197 knockdown, as detected by RT-qPCR; ${ }^{*} p<0.05,{ }^{* *} p<0.01$. Data are presented as the mean \pm S.D., and the $p$ values were determined by a two-tailed unpaired Student's $t$-test. $\mathbf{j}$ The expression of $A T P 1 B 3, B Z W 1$, and HNRNPR was detected in degenerated tissues using RT-qPCR; ${ }^{*} p<0.05$, *** $p<0.001$. Data are presented as the mean \pm S.D., and the $p$ values were determined by a two-tailed unpaired Student's $t$-test. $\mathbf{k}$ The binding site of miR1197 and ATP1B3 predicted by TargetScan.

expression. Therefore, an integrated microarray study (GSE67566) was performed on five control samples and five degeneration samples (Fig. 1d) ${ }^{22}$. In accordance with previous studies, circRNAs with significant differences between the control and degeneration groups $(\log \mathrm{FC}<-1$ or $\log \mathrm{FC}>1, p<0.05)$ were selected to generate a heat map (Fig. 1e). circRNAs that exhibited significantly lower levels in the degenerative group might have a protective effect on disc degeneration; thus, 15 circRNAs with decreased expression in the heat map were selected. To verify the heat map results, patients with fractures were selected as the control group, and three patients with different degrees of lesions were selected according to the Pfirrmann classification ${ }^{23}$. These samples were then analyzed using real-time (RT) quantitative polymerase chain reaction ( $\mathrm{qPCR}$ ), and the expression level of hsa_circ_0035875 was found to decrease with increasing lesion severity (Fig. 1f; Supplementary Fig. 1a). This suggested a protective effect of hsa_circ_0035875, which is a loop formed by exons 2-3 of the SPG21 gene (circSPG21), on degeneration of the intervertebral disc.

Three representative from the spinal fracture samples group and three samples from the intervertebral disc degeneration group were selected for analysis. The expression levels of SPG21 and circSPG21 were then measured in the samples using RT-qPCR. The results showed that the decrease in circSPG21 levels was greater than the decrease in SPG21 levels in degenerative tissues, which excluded an effect of SPG21 on circSPG21 (Fig. 1g, h). RNA fluorescence in situ hybridization (FISH) showed a decreased fluorescence intensity in the degenerated group (Fig. 1i). Therefore, we next explored the role and mechanism of circSPG21 in the intervertebral disc.

\section{circSPG21 regulates NPC extracellular matrix (ECM) metabolism}

Small-interfering RNA was transfected into NPCs to inhibit the expression of circSPG $21^{24}$, and NPCs were also infected with adenovirus $^{25}$ carrying the circSPG21 plasmid; circSPG21 expression was increased or decreased accordingly (sicircSPG21\#1\&2 were selected; Fig. 1j, k). When circSPG21 expression was suppressed, SPG21 expression was not affected, and an influence of circSPG21 on SPG21 was thus ruled out (Fig. 1l). The $\beta$-galactosidase staining results showed that circSPG21 effectively inhibited the senescence of NPCs (Fig. 1m). CircSPG21 also inhibited the degradation of the ECM based on Alcian blue staining (Fig. 1n). Additionally, western blotting revealed that the inhibition of circSPG21 expression promoted the expression of matrix metallopeptidase (MMP) 3, MMP13, ADAM metallopeptidase with thrombospondin type 1 (ADAMTS) motif 4, and ADAMTS5 and reduced the expression of collagen II, aggrecan, and SRY-box transcription factor 9 (SOX9). Increasing the expression of circSPG21 yielded the opposite results (Fig. 10) based on immunofluorescence (IF) assays ${ }^{26}$ and RT-qPCR (Fig. 1p, q, r). These results indicated that circSPG21 inhibited catabolism and enhanced anabolism.

\section{Ring structure of circSPG21}

RNA $\mathrm{FISH}^{14}$ revealed that circSPG21 mainly localized to the cytoplasm (Fig. 2a). After actinomycin D treatment (an inhibitor of transcription), the expression of circSPG21 and SPG21 was detected at various time points $(0,6,12,24,48 \mathrm{~h})$. The half-life of circSPG21 was more than $48 \mathrm{~h}$, indicating that circSPG21 was stable in NPCs (Fig. 2b). circRNA forms a ring structure through a head-totail connection; this structure differs from that of ordinary RNA in that there is no $5^{\prime}$ hat or $3^{\prime} \operatorname{poly}(A)$ structure. To clarify this structure, the sequence at the circSPG21 junction was examined using Sanger sequencing, and it was found that circSPG21 was formed by the splicing of exons 2-3 of SPG21 (Fig. 2c). To exclude the possibility of trans-splicing ${ }^{27}$ (two different pre-mRNA exons were linked together, which has been observed in various species), a convergent primer was designed to amplify SPG21 mRNA, and a divergent primer was designed to amplify circSPG21 from both cDNA and genomic DNA (gDNA). Bands were amplified from cDNA with both primers; however, only the SPG21 band was amplified from gDNA (Fig. 2d). This evidence indicated that circSPG21 formed a stable ring structure via the end-to-end connection of SPG21 mRNA and was widely present in the cytoplasm.

\section{ceRNA network construction}

circRNA exists widely in eukaryotic cells and acts as an miRNA sponge. Using online databases (TargetScan: www.targetscan.org/; miRanda: www.miranda.org/; and RNAhybrid: RNA hybridization software), nine miRNAs that could stably bind circSPG21 were identified (Fig. 3a). The mechanistic circRNA-microRNA-target gene network was constructed using an online database (Fig. 3b). To verify the relationship between miRNA and circSPG21, the expression of circSPG21 was inhibited in NPCs, and it was found that the expression of miR-1197 significantly increased (Fig. 3c; Supplementary Fig. 1b) ${ }^{28}$. Therefore, it was hypothesized that miR-1197 might be the downstream target of circSPG21 and could play a role in disc degeneration. The expression of miR-1197 was increased in degenerative tissues, as confirmed by both RT-qPCR (Fig. 3d) and FISH (Fig. 3e) assays. FISH analyses revealed that circSPG21 and miR1197 colocalized in the cytoplasm, and there was an intersection in the cell space (Fig. 3f). The targets of the interaction between circSPG21 and miR-1197 were predicted using an online database (Fig. 3g). Therefore, miR-1197 was selected for further analysis.

Target genes of miR-1197 were predicted using three online databases (TargetScan, miRDB, and miRTarBase), and the genes common to the three prediction results were selected as the three most suitable target genes (Fig. 3h). The expression of miR-1197 was inhibited in NPCs, and it was found that among the three target genes, ATP1B3 expression significantly increased; thus, it was hypothesized that ATP1B3 might be the target gene of miR1197 (Fig. 3i). Furthermore, the expression of ATP1B3 was decreased in degenerated tissue of the intervertebral disc, and ATP1B3 was therefore selected to explore its function and 


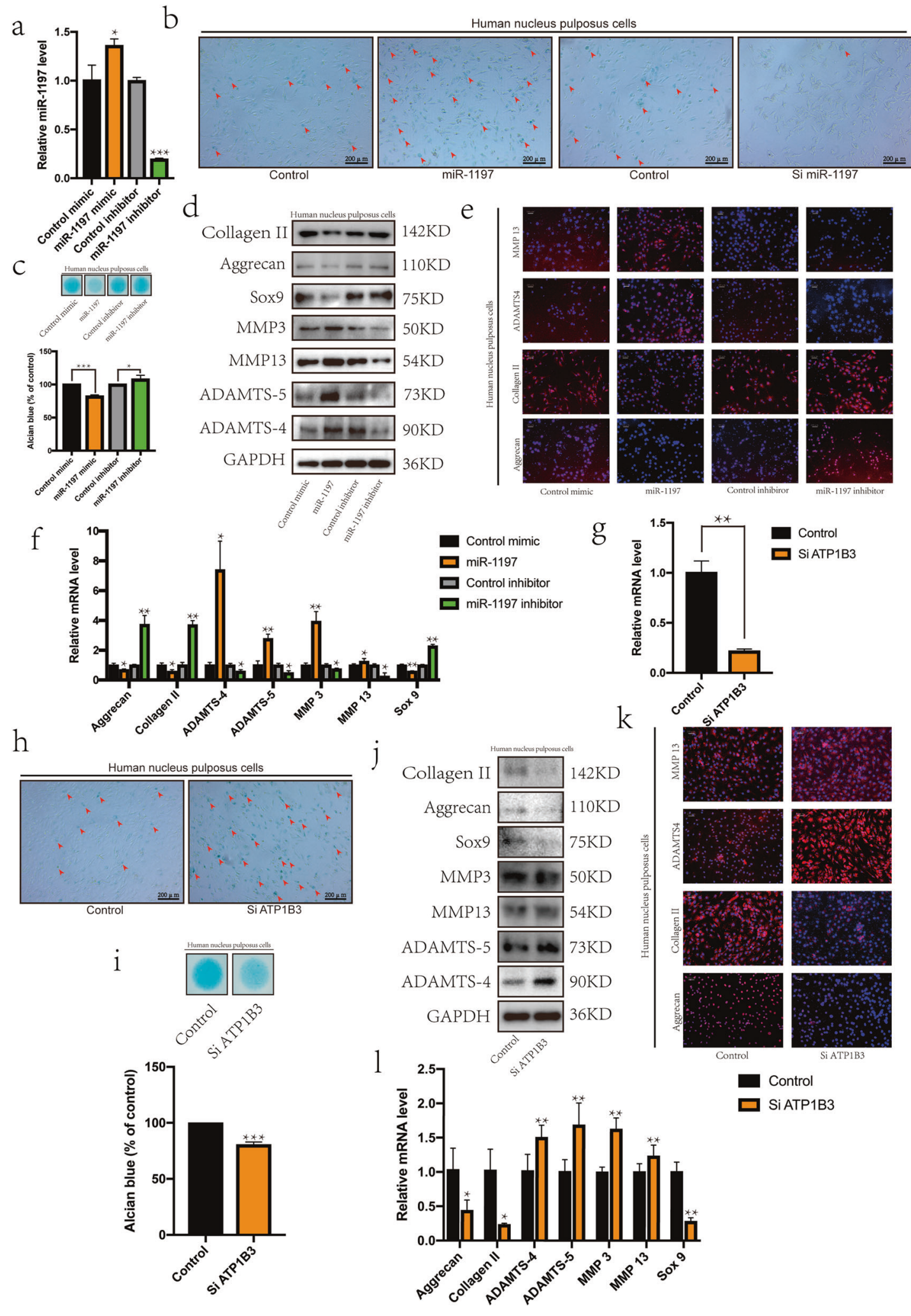

mechanism (Fig. 3j). The target sequence of miR1197 with respect to ATP1B3 was predicted (Fig. 3k).

MiR-1197 and ATP1B3 affect the activity of NPCs

To study the role of miR-1197, its expression was downregulated or upregulated in NPCs (Fig. 4a). miR-1197 accelerated the senescence of NPCs (Fig. 4b) and decomposition of the ECM (Fig. 4c). From the western blot results, it was observed that the expression of collagen II, aggrecan, and SOX9 decreased, but the expression of MMP3, MMP13, ADAMTS4, and ADAMTS5 increased, when miR-1197 was overexpressed. The opposite results were obtained when miR-1197 was knocked down (Fig. 4d). 
Fig. 4 MiR-1197 and ATP1B3 affect the activity of NPCs. a The efficiency of transfection with miR-1197 mimics and inhibitors, as determined by real-time quantitative polymerase chain reaction (RT-qPCR); ${ }^{*}<0.05,{ }^{* * *} p<0.001$. Data are presented as the mean \pm S.D., and the $p$ values were determined by a two-tailed unpaired Student's $t$-test. $\mathbf{b} \beta$-Galactosidase staining was performed to evaluate cell aging (scale bar, $200 \mu \mathrm{m}$ ). c Alcian staining was performed to evaluate the extracellular matrix; ${ }^{*} p<0.05,{ }^{* *} p<0.01$. Data are presented as the mean \pm S.D., and the $p$ values were determined by a two-tailed unpaired Student's t-test. d After the expression of miR-1197 was knocked down or miR-1197 was overexpressed, the expression of related proteins (collagen II, aggrecan, SRY-box transcription factor 9 (SOX9), matrix metallopeptidase 3 (MMP3), MMP13, ADAM metallopeptidase with thrombospondin type 1 motif 4 (ADAMTS4), and ADAMTS5) was detected by western blotting. e Expression of collagen II, aggrecan, ADAMTS4, and MMP13 was measured using immunofluorescence (IF) comparing the fluorescence intensity (scale bar, $100 \mu \mathrm{m}$ ). $\mathbf{f}$ Expression of related genes, as detected by RT-qPCR; ${ }^{*} p<0.05,{ }^{* *} p<0.01$. Data are presented as the mean \pm S.D., and the $p$ values were determined by a two-tailed unpaired Student's $t$-test. $\mathbf{g}$ The efficiency of siRNA targeting ATP1B3 was tested using RTqPCR; ${ }^{* *} p<0.01$. Data are presented as the mean \pm S.D., and the $p$ values were determined by a two-tailed unpaired Student's $t$-test. h $\beta$-Galactosidase staining was performed to evaluate cell aging (scale bar, $200 \mu \mathrm{m}$ ). i Alcian staining was performed to evaluate the extracellular matrix. ${ }^{*} p<0.05,{ }^{* *} p<0.01$. Data are presented as the mean \pm S.D., and the $p$ values were determined by a two-tailed unpaired Student's $t$-test. j Expression of proteins (collagen II, aggrecan, SRY-box transcription factor 9 (SOX9), matrix metallopeptidase 3 (MMP3), MMP13, ADAM metallopeptidase with thrombospondin type 1 motif 4 (ADAMTS4), and ADAMTS5) after knocking down ATP1B3, as determined by western blotting. k Fluorescence intensity of proteins (collagen II, aggrecan, ADAMTS4, and MMP13) after the knockdown of $A T P 1 B 3$, as determined by immunofluorescence (scale bar, $100 \mu \mathrm{m}$ ). I Expression of genes after the knockdown of ATP1B3, as detected by RTqPCR; ${ }^{*} p<0.05,{ }^{* *} p<0.01$. Data are presented as the mean \pm S.D., and the $p$ values were determined by a two-tailed unpaired Student's $t$-test.

IF and RT-qPCR both showed similar results (Fig. 4e, f). Therefore, circSPG21 can affect ECM metabolism by NPCs through miR-1197.

Next, the efficiency of ATP1B3 siRNA was tested (Fig. 4g). When ATP1B3 expression was decreased, NPCs displayed accelerated senescence (Fig. 4h) and ECM decomposition (Fig. 4i), indicating that ATP1B3 exerts a protective effect on NPCs. In addition, the expression of collagen II, aggrecan, and SOX9 decreased, whereas the expression of MMP3, MMP13, ADAMTS4, and ADAMTS5 increased, as observed by western blotting (Fig. 4j) and IF (Fig. 4k). Similar results were observed using RT-qPCR (Fig. 4l). These results indicated that ATP1B3 had a protective effect on NPCs.

\section{Verification of the circSPG21/miR-1197/ATP1B3 axis in NPCs} To determine whether the function of circSPG21 affects IVDD via miR-1197, a rescue experiment was designed in which the results of knocking down circSPG21 expression alone were compared with those of knocking down circSPG21 and miR-1197 expression at the same time. The knockdown of miR-1197 by decreasing the expression of circSPG21 successfully rescued the aging trend of NPCs (Fig. 5a) and inhibited the decomposition of the ECM (Fig. 5b). Similarly, when cells were transfected with circSPG21 alone, matrix-synthesizing proteins (collagen II, aggrecan, and SOX9) were inhibited, and the expression of degrading enzymes (MMP3, MMP13, ADAMTS4, and ADAMTS5) was promoted. However, these results were reversed when circSPG21 and miR-1197 were cotransfected (Fig. 5c). The IF and RT-qPCR results also showed that the cotransfection of circSPG21 and miR-1197 salvaged gene and protein expression (Fig. 5d, e). In conclusion, as a downstream molecule of circSPG21, miR-1197 aggravates NP degeneration.

Next, the relationship between miR-1197 and ATP1B3 was explored using a rescue experiment. The knockdown of ATP1B3 (based on the low expression of miR-1197) accelerated the aging trend of NPCs (Fig. 5f) and the decomposition of the ECM (Fig. 5g). Similarly, when miR-1197 expression decreased, the expression of collagen II, aggrecan, and SOX9 increased, whereas the expression of MMP3, MMP13, ADAMTS4, and ADAMTS5 decreased at both the gene and protein levels. Cotransfection with si-miR-1197 and si-ATP1B3 yielded the opposite expression trend (Fig. 5h-j). These results indicated that ATP1B3 is the downstream effector of miR-1197.

\section{Injection of circSPG21 alleviates IVDD in a mouse model}

As shown in Fig. 6a, the tail of a mouse was fixed to form a ring by penetrating the mouse tail bone with a thin wire; thus, the pressure on the intervertebral disc tissue in the tails of the mice was increased. Under pressure, intervertebral disc tissue degenerates. Six-week-old male mice were selected, and their tails were fixed for two months to form a tail-looping model ${ }^{18}$. Then, the experimental group was intraperitoneally injected with adenovirus carrying the circSPG21 plasmid, and the control group was injected with normal saline once per week for eight weeks (Fig. 6b). The tails were subsequently removed, and radiographs were taken. The caudate bone in the tail-looping model mice was morbidly angulated, and the disc height index was decreased, indicating compression of the tail disc (Fig. 6c). circSPG21 and ATP1B3 expression was increased in mice injected with the circSPG21-wt plasmid, as measured by RT-qPCR (Fig. 6d, e). The tails of the mice were then paraffin sectioned and stained with $\mathrm{HE}$, safranin-O/fast green, and toluidine blue. Relative to normal tissue, the degenerative disc tissue showed a loss of NP tissue (Fig. 6f). Total protein was next extracted from the tail intervertebral disc tissue for western blotting (Fig. $6 \mathrm{~g}$ ). The results showed that anabolic activity decreased and catabolism increased in degenerative tissues. However, increased circSPG21 expression changed this trend and protected the NP; this result was confirmed by immunohistochemistry (Fig. 6h).

\section{DISCUSSION}

The development of new treatment alternatives for IVDD has been stagnating; thus, it is imperative to explore its pathogenesis to identify new targets. circRNAs are widely expressed in mammalian cells, show tissue specificity, have a conserved and stable structure, and offer great potential for the development of new treatments. Several studies have shown that circRNAs are associated with human diseases. However, little is known about their mechanism in the occurrence and development of IVDD, which suggests that this should be explored ${ }^{8}$.

In this study, it was first determined that circSPG21 is the key circRNA involved in IVDD. circSPG21 was determined to be involved in the synthesis and degradation of IVDD-related proteins. The potential impact of circSPG21 on the regulatory function of miRNAs in NPCs was then studied. circSPG21 significantly reduced activity and function by capturing miR1197. Therefore, it was hypothesized that miR-1197 was a downstream target molecule of circSPG21 that promoted anabolism and inhibited catabolism. Furthermore, the overexpression of ATP1B3 induced by miR-1197 gene knockout exerted a protective effect on IVDD. Therefore, a prospective strategy of targeting the circSPG21/miR-1197/ATP1B3 axis for IVDD treatment was proposed (Fig. 7). Previous studies have shown that intervertebral disc degeneration is closely related to inflammation. However, we identified downstream target genes that suggested that intervertebral disc degeneration might be related to the intracellular 
a

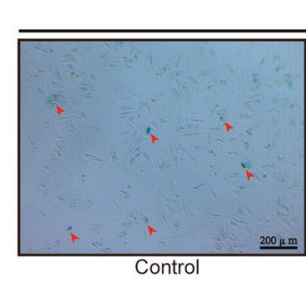

Human nucleus pulposus cells

C

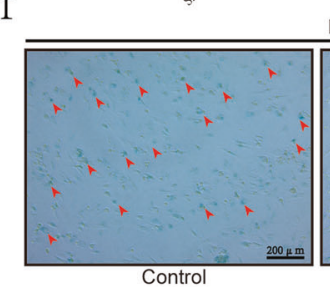

$\mathrm{h}$

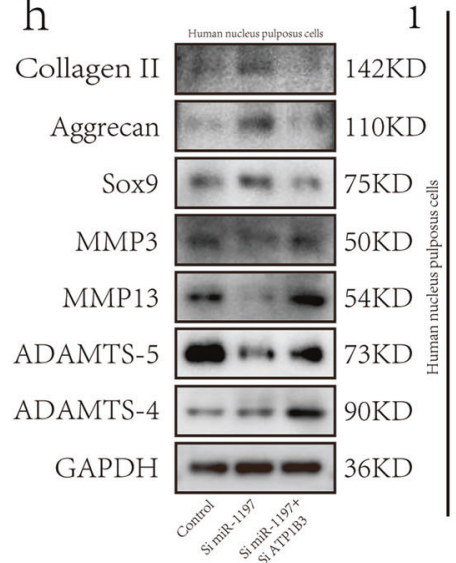

d

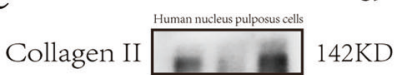

Aggrecan $110 \mathrm{KD}$

Sox 9

MMP3

MMP13

ADAMTS-5 $+4=$

ADAMTS-4

GAPDH

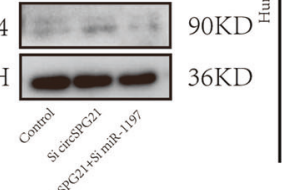

Human nucleus pulposus cells
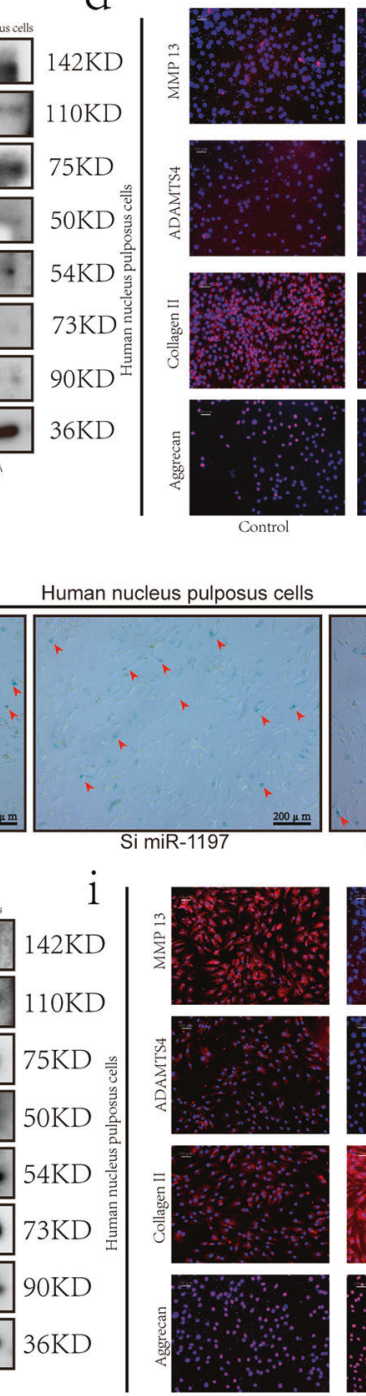

Control
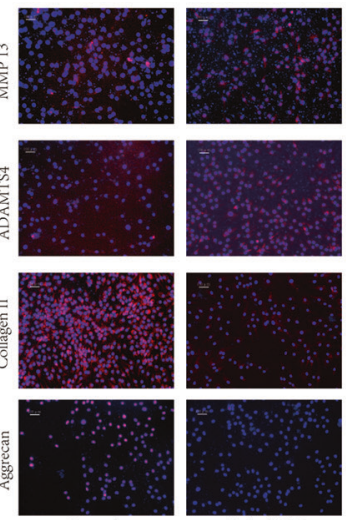

Si circSPG21
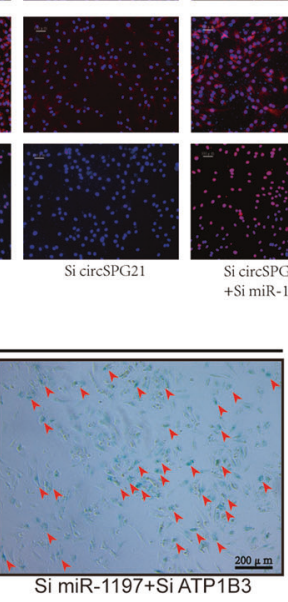
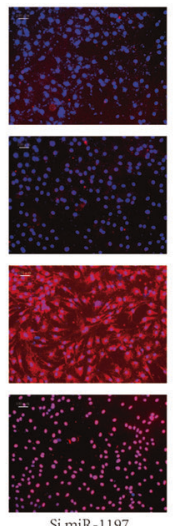
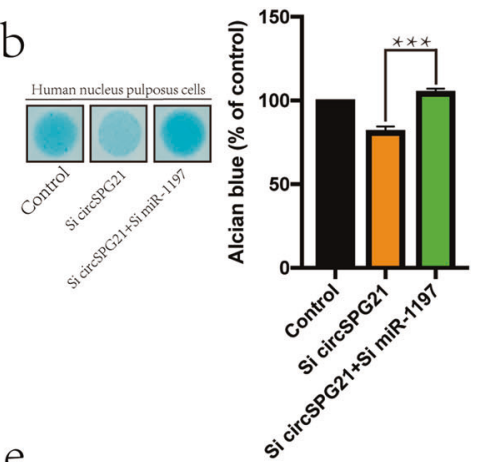

e
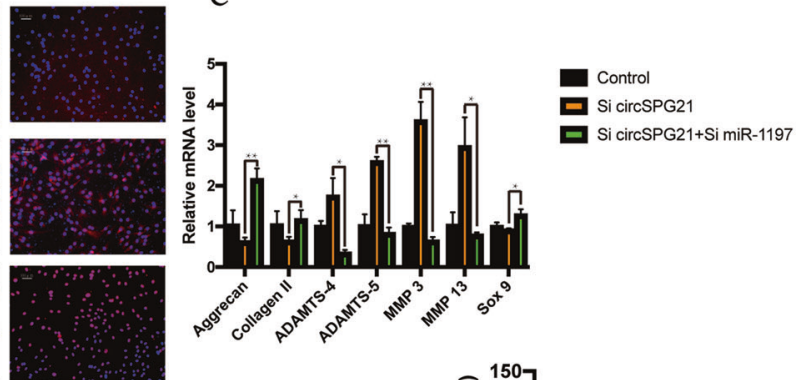

Si circSPG21
+ Si miR-1197

g
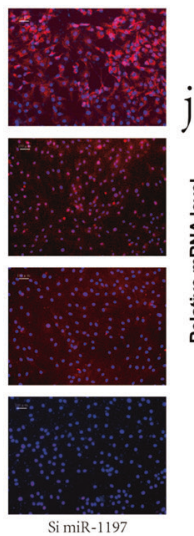

Si miR-1197
+ +Si ATP1B3
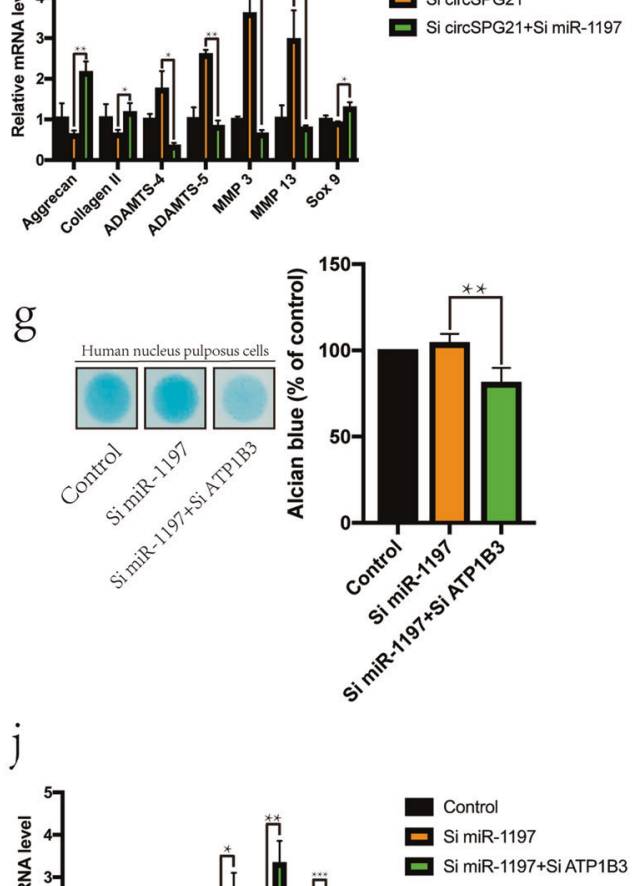

Fig. 5 Verification of the circSPG21/miR-1197/ATP1B3 axis in NPCs. a $\beta$-galactosidase staining was performed to evaluate cell aging (scale bar, $200 \mu \mathrm{m})$. b Alcian staining was performed to evaluate the extracellular matrix; ${ }^{*} p<0.05,{ }^{* *} p<0.01$. Data are presented as the mean \pm S.D., and the $p$ values were determined by a two-tailed unpaired Student's $t$-test. c Changes in the expression of proteins (collagen II, aggrecan, SRY-box transcription factor 9 (SOX9), matrix metallopeptidase 3 (MMP3), MMP13, ADAM metallopeptidase with thrombospondin type 1 motif 4 (ADAMTS4), and ADAMTS5) when circSPG21 was knocked down, either alone or in combination with miR-1197. $\mathbf{d}$ Fluorescence intensity of collagen II, aggrecan, ADAMTS4, and MMP13 based on immunofluorescence (IF; scale bar, $100 \mu \mathrm{m}$ ). e Expression of genes (collagen II, aggrecan SOX9, MMP3, MMP13, ADAMTS4, and ADAMTS5), as detected by real-time quantitative polymerase chain reaction (RT-qPCR); ${ }^{*}<$ $0.05,{ }^{* *} p<0.01$. Data are presented as the mean \pm S.D., and the $p$ values were determined by a two-tailed unpaired Student's $t$-test. f $\beta$-Galactosidase staining was performed to evaluate cell aging (scale bar, $200 \mu \mathrm{m}$ ). g Alcian staining was performed to evaluate the extracellular matrix. ${ }^{*} p<0.05,{ }^{* *} p<0.01$. Data are presented as the mean \pm S.D., and the $p$ values were determined by a two-tailed unpaired Student's t-test. $\mathbf{h}$ Expression of proteins (collagen Il, aggrecan SOX9, MMP3, MMP13, ADAMTS4, and ADAMTS5) when miR-1197 was knocked down, either alone or in combination with ATP1B3, as determined by western blotting. i Fluorescence intensity of proteins (collagen II, aggrecan, ADAMTS4, and MMP13) when miR-1197 was knocked down, either alone or in combination with ATP1B3, as determined by IF (scale bar, $100 \mu \mathrm{m}$ ). j Expression of genes when miR-1197 was knocked down, either alone or in combination with ATP1B3, as detected by RTqPCR; ${ }^{*} p<0.05,{ }^{* *} p<0.01,{ }^{* *} p<0.001$. Data are presented as the mean \pm S.D., and the $p$ values were determined by a two-tailed unpaired Student's $t$-test. 

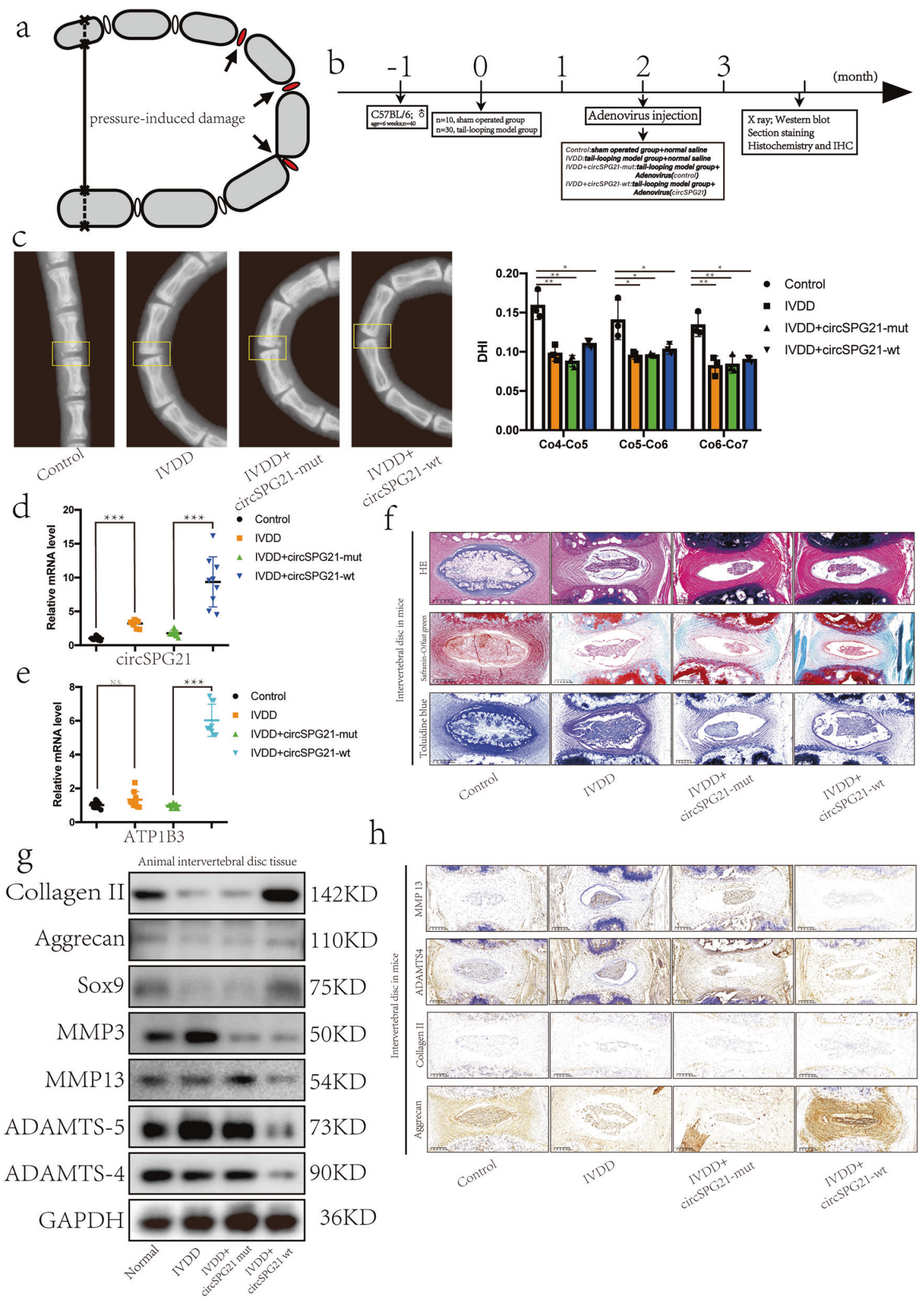

ion concentration. ATP1B3 encodes an $\mathrm{Na}^{+} / \mathrm{K}^{+}$ion channel protein in the cell membrane. In degenerative tissues, decreased ATP1B3 expression prevents the exchange of $\mathrm{Na}^{+} / \mathrm{K}^{+}$ions. We hypothesize that $\mathrm{Na}^{+} / \mathrm{K}^{+}$ions accumulate in cells in degenerated tissues, intracellular osmotic pressure rises, and water passively enters cells, causing a deformed cell morphology. However, the relationship between the changes in ion concentrations and IVDD warrants further study.

To date, research on the ceRNA network has shown that the interacting factors in the network are closely related to the occurrence and development of human diseases. circWHSC1 is considerably more highly expressed in endometrial carcinoma than 
Fig. 6 Injection of circSPG21 alleviates IVDD in a mouse model. a Schematic diagram showing the composition and principle of the taillooping model. b Diagram showing the time sequence of in vivo experiments. $\mathbf{c}$ Left, the tails of mice, as observed by X-ray. Right panel, disc height index (DHI) of coccyx4-coccyx5, coccyx5-coccyx6, and coccyx7-coccyx8; $n=12,{ }^{*} p<0.05,{ }^{* *} p<0.01$. Data are presented as the mean \pm S.D., and the $p$ values were determined by a two-tailed unpaired Student's $t$-test. $\mathbf{d}$ Expression of circSPG21 in the intervertebral discs of mice after the injection of adenovirus or saline, as detected by real-time quantitative polymerase chain reaction (RT-qPCR); $n=12, * * * p<0.001$. Data are presented as the mean \pm S.D., and the $p$ values were determined by a two-tailed unpaired Student's $t$-test. e Expression of ATP1B3 in the intervertebral disc of mice, as detected by RT-qPCR; $n=12,{ }^{* *} p<0.001$. Data are presented as the mean \pm S.D., and the $p$ values were determined by a two-tailed unpaired Student's $t$-test. $\mathrm{f}$ Tissue sections of mice stained with hematoxylin and eosin (HE), safranin-O/fast green, and toluidine blue to show the degree of intervertebral disc degeneration (scale bar, $200 \mu \mathrm{m}$ ). $\mathbf{g}$ Expression of collagen II, aggrecan, SRY-box transcription factor 9 (SOX9), matrix metallopeptidase 3 (MMP3), MMP13, ADAM metallopeptidase with thrombospondin type 1 motif 4 (ADAMTS4), and ADAMTS5 in intervertebral disc tissue, as determined by western blotting. $\mathbf{h}$ Expression of proteins (collagen II, aggrecan, ADAMTS4, and MMP13), as determined by immunohistochemical staining (scale bar, $200 \mu \mathrm{m}$ ).

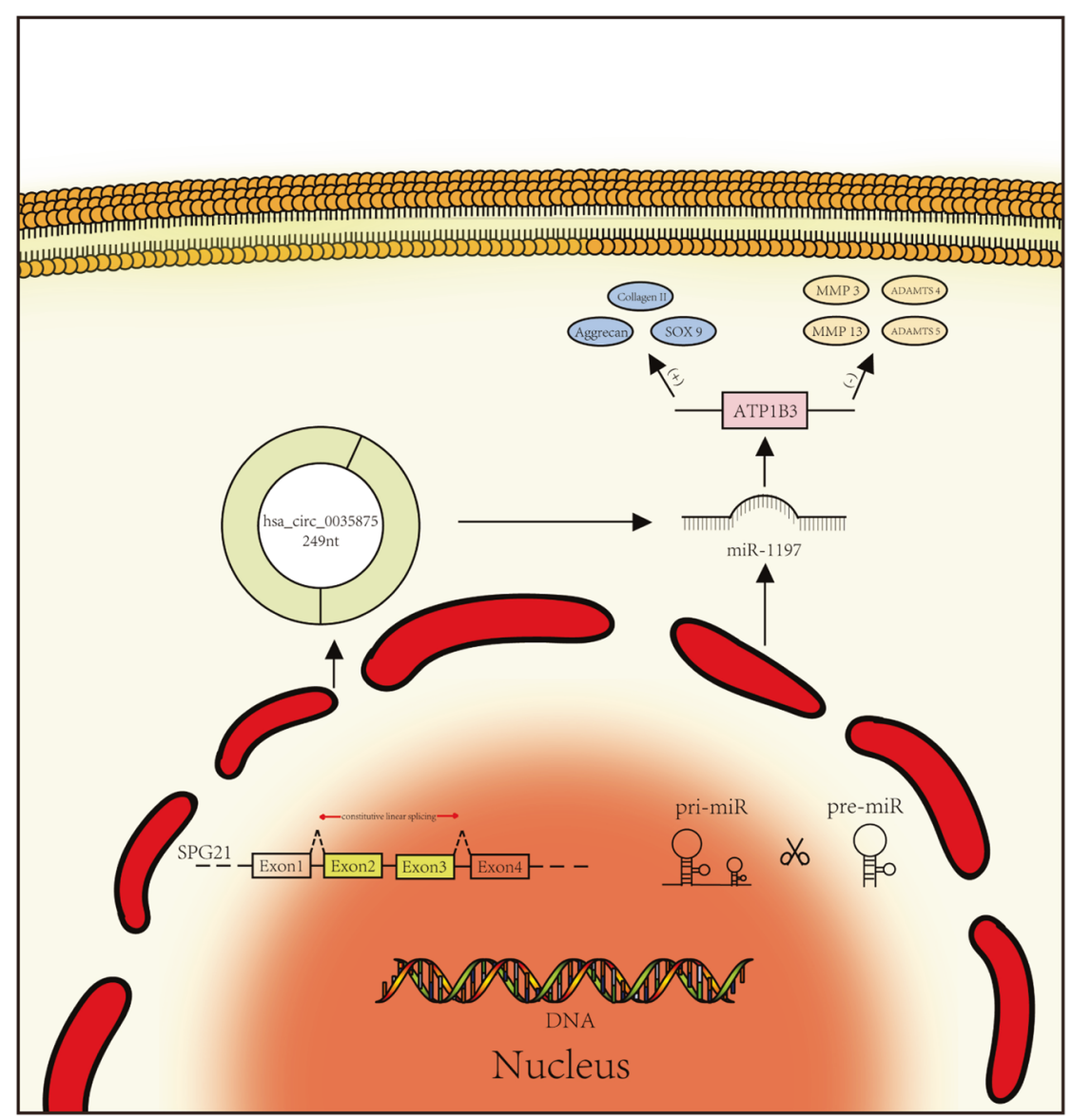

Fig. 7 Schematic diagram of circSPG21 mechanism. A schematic diagram of the mechanism underlying the effect of circular RNA.

in normal tissues. Through bioinformatics prediction and experimental verification, the circWHSC1/miR-646/NPM1 axis was found to promote the development of endometrial cancer ${ }^{29}$. Through the microarray analysis of metastatic and nonmetastatic human hepatocellular carcinoma tissues, the IncRNA CDKN2BAS was found to be significantly upregulated in metastatic tumors. With expanding research, CDKN2BAS was determined to regulate miR-153-5p and act as a ceRNA, which could increase the expression of $A R H G A P 18$, the target gene of miR-153-5 $\mathrm{p}^{30}$. The ceRNA network is a platform that modulates protein-encoding mRNA and nonencoding RNA and might represent a wide range of posttranscriptional regulatory events related to gene expression involved in physiology and pathology. With accumulating research, ceRNA network interactions could be exploited in RNA-directed therapy.

However, there are limitations to this study. Age differences between patients with and without IVDD might have led to some bias. Moreover, because of the clinicopathological characteristics of IVDD, the detection of NP tissue is an inevitable confounding factor.
Therefore, animal model experiments were conducted with the hope that this approach would help eliminate the influence of age and individual factors. In addition to its ceRNA function, there could be other potential mechanisms through which circSPG21 regulates IVDD, such as by interacting with RNA-binding proteins ${ }^{31}$. Furthermore, the expression level of circSPG21 decreased during the degeneration of NPCs. We speculate that there is a complex regulatory mechanism upstream of circSPG21. The specific mechanism of circSPG21 thus still needs to be further explored. In conclusion, circSPG21 reduces the apoptosis of NPCs induced by inflammatory cytokines and the imbalance of ECM anabolism and catabolism through miR-1197/ATP1B3. Thus, targeting circSPG21 may provide an effective treatment strategy for IVDD.

\section{DATA AVAILABILITY}

All data included in this study are available upon request by contacting the corresponding author. 


\section{REFERENCES}

1. Gautschi, O. P. et al. Influence of age on pain intensity, functional impairment and health-related quality of life before and after surgery for lumbar degenerative disc disease. Clin. Neurol. Neurosurg. 150, 33-39 (2016).

2. Chuah, Y. J. et al. Development of annulus fibrosus tissue construct with hydrogel coils containing pre-conditioned mesenchymal stem cell. J. Mater. Sci. Technol. 63, 27-34 (2020).

3. Brotzki, C. et al. Comparison of different hybrid techniques for the treatment of multilevel cervical degenerative disc disease-analysis of prospectively collected clinical, radiologic, and psychological parameters. World Neurosurg. 140, e112-e120 (2020).

4. Hodgkinson, T. et al. Therapeutic potential of growth differentiation factors in the treatment of degenerative disc diseases. Jor. Spine 2, e1045 (2019).

5. Schnack, N. B., Trine, M. \& Jørgen, K. Automated one-double-Z pair BaseScope ${ }^{\text {TM }}$ for CircRNA in situ hybridization. Methods Mol. Biol. 2148, 379-388 (2020).

6. Jingwei, Z. et al. A circRNA derived from linear HIPK3 relieves the neuronal cell apoptosis in spinal cord injury via ceRNA pattern. Biochem. Biophys. Res. Commun. 528, 359-367 (2020).

7. Eringyte, I. et al. Coordinated $A R$ and microRNA regulation in prostate cancer. Asian J. Urol. 7, 233-250 (2020).

8. Cheng, $X$. et al. Circular RNA VMA21 protects against intervertebral disc degeneration through targeting miR-200c and $X$ linked inhibitor-of-apoptosis protein. Ann. Rheum. Dis. 77, 770-779 (2018).

9. Xie, L. et al. CircERCC2 ameliorated intervertebral disc degeneration by regulating mitophagy and apoptosis through miR-182-5p/SIRT1 axis. Cell Death Dis. 10, 751 (2019).

10. Lan-Fang, S., Xue-Jiao, C. \& Zi-Bing, J. Emerging roles of noncoding RNAs in retinal diseases: a review. Clin. Exp. Ophthalmol. 48, 1085-1101 (2020).

11. Sun, Y., Wang, X., Fu, G. \& Geng, X. MicroRNA-199a-5p accelerates nucleus pulposus cell apoptosis and IVDD by inhibiting SIRT1-mediated deacetylation of p21. Mol. Ther. Nucleic Acids 24, 634-645 (2021).

12. Wenhua, J. et al. MiR-532-3p inhibits metastasis and proliferation of non-small cell lung cancer by targeting FOXP3. J. Buon. 24, 2287-2293 (2019).

13. Cao, M. L. et al. MicroRNA-144 regulates cardiomyocyte proliferation and apoptosis by targeting TBX1 through the JAK2/STAT1 pathway. Cytogenet. Genome Res. 159, 190-200 (2019).

14. Aughey, R. J. et al. Muscle $\mathrm{Na}^{+}-\mathrm{K}^{+}$-ATPase activity and isoform adaptations to intense interval exercise and training in well-trained athletes. J. Appl. Physiol. (1985) 103, 39-47 (2007).

15. Scarlato, M. et al. Exome sequencing reveals a novel homozygous mutation in ACP33 gene in the first Italian family with SPG21. J. Neurol. 264, 2021-2023 (2017).

16. Baisong, Z. et al. ATP1B3 cooperates with BST-2 to promote hepatitis B virus restriction. J. Med. Virol. 92, 201-209 (2020).

17. Sylvia, H. et al. Proliferation, migration, and ECM formation potential of human annulus fibrosus cells is independent of degeneration status. Cartilage 11, 192-202 (2020).

18. Nakamichi, R. et al. Mohawk promotes the maintenance and regeneration of the outer annulus fibrosus of intervertebral discs. Nat. Commun. 7, 12503 (2016).

19. Moon, S. H. et al. Human intervertebral disc cells are genetically modifiable by adenovirus-mediated gene transfer: implications for the clinical management of intervertebral disc disorders. Spine (Philos. Pa 1976) 25, 2573-2579 (2000).

20. Debacq-Chainiaux, F., Erusalimsky, J. D., Campisi, J. \& Toussaint, O. Protocols to detect senescence-associated beta-galactosidase (SA-betagal) activity, a biomarker of senescent cells in culture and in vivo. Nat. Protoc. 4, 1798-1806 (2009).

21. Nishida, Y., Knudson, C. B. \& Knudson, W. Osteogenic protein-1 inhibits matrix depletion in a hyaluronan hexasaccharide-induced model of osteoarthritis. Osteoarthr. Cartil. 12, 374-382 (2004).

22. Lan, P. H. et al. Landscape of RNAs in human lumbar disc degeneration. Oncotarget 7, 63166-63176 (2016).

23. Vedran, M. et al. Level of education as a risk factor for extensive prevalence of cervical intervertebral disc degenerative changes and chronic neck pain. Cent. Eur. J. Public Health 25, 245-250 (2017).

24. Kim, Y., Kim, H. S., Sohn, J. \& Ji, J. D. 1,25-Dihydroxyvitamin D 3 induces human myeloid cell differentiation via the mTOR signaling pathway. Biochem. Biophys. Res. Commun. 519, 909-915 (2019).

25. Chisa, $H$. et al. Acceleration of cartilage repair by genetically modified chondrocytes over expressing bone morphogenetic protein-7. J. Orthop. Res. 21, 573-583 (2003).
26. Dai, P. et al. Validation of phospholipase A 2 receptor direct immunofluorescence staining in the diagnosis of primary membranous glomerulonephritis. Pathology 52, 546-551 (2020).

27. Pervouchine, D. D. Circular exonic RNAs: when RNA structure meets topology. Biochim. Biophys. Acta Gene Regul. Mech. 1862, 194384 (2019).

28. Sun, B. et al. MicroRNA-1197 downregulation inhibits proliferation and migration in human non- small cell lung cancer cells by upregulating HOXC11. Biomed. Pharmacother. 117, 109041 (2019).

29. Liu, Y. et al. CircRNA WHSC1 targets the miR-646/NPM1 pathway to promote the development of endometrial cancer. J. Cell. Mol. Med. 24, 6898-6907 (2020).

30. Chen, J. et al. LncRNA CDKN2BAS predicts poor prognosis in patients with hepatocellular carcinoma and promotes metastasis via the miR-153-5p/ ARHGAP18 signaling axis. Aging (Albany NY) 10, 3371-3381 (2018).

31. Chen, R. X. et al. N(6)-methyladenosine modification of circNSUN2 facilitates cytoplasmic export and stabilizes HMGA2 to promote colorectal liver metastasis. Nat. Commun. 10, 4695 (2019).

\section{ACKNOWLEDGEMENTS}

This research was supported by the National Nature Science Fund of China (81972504, 81802680), the Zhejiang Provincial Natural Science Foundation of China (LY19H160058), and the Medical Healthy Scientific Technology Project of Zhejiang Province (2020388803, 2020RC070). No benefits in any form have been or will be received from any commercial party related directly or indirectly to the subject of this manuscript.

\section{AUTHOR CONTRIBUTIONS}

J.M. and S.S. conceived and supervised the study; Y.H. designed the experiments; Y.H., J.G., J.W. and H.Y. performed the experiments; T.Y. provided new tools and reagents; Y.X. developed new software and performed simulation studies; H.Y. analyzed the data; H.Y. and Z.C. wrote the manuscript; J.M. and S.S. revised the manuscript.

\section{COMPETING INTERESTS}

The authors declare no competing interests.

\section{ADDITIONAL INFORMATION}

Supplementary information The online version contains supplementary material available at https://doi.org/10.1038/s12276-021-00674-z.

Correspondence and requests for materials should be addressed to Bin Fang or Jianjun Ma.

Reprints and permission information is available at http://www.nature.com/ reprints

Publisher's note Springer Nature remains neutral with regard to jurisdictional claims in published maps and institutional affiliations.

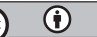

Open Access This article is licensed under a Creative Commons Attribution 4.0 International License, which permits use, sharing, adaptation, distribution and reproduction in any medium or format, as long as you give appropriate credit to the original author(s) and the source, provide a link to the Creative Commons license, and indicate if changes were made. The images or other third party material in this article are included in the article's Creative Commons license, unless indicated otherwise in a credit line to the material. If material is not included in the article's Creative Commons license and your intended use is not permitted by statutory regulation or exceeds the permitted use, you will need to obtain permission directly from the copyright holder. To view a copy of this license, visit http://creativecommons. org/licenses/by/4.0/.

(c) The Author(s) 2021 